\title{
Clinical and pulmonary function changes in cough variant asthma with small airway disease
}

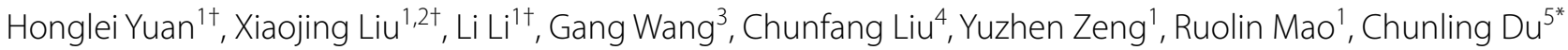
and Zhihong Chen ${ }^{1 *}$ (D)

\begin{abstract}
Background: It is known that small airway disease is present across all asthma severities; however, its prevalence and clinical characteristics in cough variant asthma (CVA) have not been fully illuminated.

Methods: A total of 77 CVA patients with preserved proximal airway function (FEV1/FVC $>70 \%$ ) were enrolled in this study. The correlation between forced expiratory flow at 50\% ( $\left.\mathrm{FEF}_{50 \%}\right)$ and $\mathrm{FEF}_{25-75 \%}$ in the CVA population was first evaluated. $\mathrm{FFF}_{50 \%}$ was determined to be an easy and feasible parameter for identifying small airway disease. CVA with

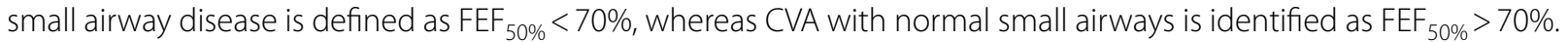
Demographic features, clinical characteristics, lung function and induced sputum test results were determined at the initial visit and at the final visit 1 year later.

Results: $\mathrm{FEF}_{50 \%}$ is a good marker for small airway disease. The cutoff value of $70 \%$ is more sensitive than the previously published $60 \%$ for identifying more patients with small airway problems early. Nearly half of the CVA population (45.4\%) in our cohort had small airway disease. In both group, symptoms improved greatly after antiasthmatic treatment. Interestingly, the changes in symptom scores [Asthma Control Test (ACT) and ACQ] were even greater in the CVA with small airway disease group than in the control group because of the higher medication usage in this subpopulation in real life. However anti-asthmatic therapy can not reverse small airway dysfunction. At last visit, $\mathrm{FEF}_{50 \%}$ of CVA with small airway diseases was $57.2 \% \pm 10.5 \%$, still much lower than the control group $\left(\mathrm{FEF}_{50 \%}=92.6 \% \pm 16.5 \%\right)$.
\end{abstract}

Conclusions: In our cohort, nearly half of the CVA population had small airway disease. Their demographic features, clinical characteristics, airway eosinophils and drug responsiveness were quite similar between two groups, which means these indices can not be used as markers to identify small airway obstruction. We found $\mathrm{FEF}_{50 \%}$ is an easy and feasible marker for early identification. Regular anti-asthmatic medication helped to improve clinical scores in patients with small airway disease, but the obstruction could not be reversed over 1-year period.

Keywords: Cough variant asthma, Small airway disease, Forced expiratory flow at 50\% ( $\left.\mathrm{FEF}_{50 \%}\right)$

\footnotetext{
*Correspondence: czh60@hotmail.com; duchunling966@163.com

${ }^{\dagger}$ Honglei Yuan, Xiaojing Liu and Li Li contributed equally to this paper

${ }^{1}$ Respiratory Division of Zhongshan Hospital, Shanghai Institute

of Respiratory Disease, Fudan University, No. 180 Fenglin Road, Shanghai,

China

${ }^{5}$ Respiratory Division of Qingpu Hospital Affiliated to Zhongshan

Hospital, Fudan University, Shanghai, China

Full list of author information is available at the end of the article
}

(c) The Author(s) 2019. This article is distributed under the terms of the Creative Commons Attribution 4.0 International License (http://creativecommons.org/licenses/by/4.0/), which permits unrestricted use, distribution, and reproduction in any medium, provided you give appropriate credit to the original author(s) and the source, provide a link to the Creative Commons license, and indicate if changes were made. The Creative Commons Public Domain Dedication waiver (http://creativecommons.org/ publicdomain/zero/1.0/) applies to the data made available in this article, unless otherwise stated. 


\section{Background}

The small airways are those with an internal diameter less than $2 \mathrm{~mm}$. They extend from the 8th generation airways to the alveoli. The total cross-sectional surface area of small airways is much greater than that of large airways; however, it only contributes to $10-29 \%$ of total airway resistance $[1,2]$. That is why small airways are referred to as a "silent zone" where chronic disease can accumulate over many years without being noticed [3, 4].

Recently, increasing evidence indicates that inflammatory infiltration and functional impairment affect not only large airways but also small airways in asthmatics $[5,6]$. In a systematic review, Usmani et al. [7] examined 15 studies of small airway diseases in adult asthma; they found that the overall prevalence of small airway disease was approximately $50-60 \%$ and that it was present across all asthma severities. In severe asthma, Berge et al. [8] found that inflammatory processes and mucus plugging were present in both large and small airways. Furthermore, high-resolution computerized tomography (HRCT) scanning has shown that small airway disease is also present in milder asthma, likely because of greater air trapping in mild disease.

Cough variant asthma (CVA) is a distinct asthma subset in which the only respiratory symptom is chronic cough. It shares the pathophysiological features of bronchial hyperresponsiveness and eosinophilic infiltration with classic asthma but is relatively milder in severity [9]. In this study, the proportion of small airway disease in CVA and its clinical characteristics, treatment regimen and lung function outcomes were observed for a year. Forced expiratory flow at $50 \%\left(\mathrm{FEF}_{50 \%}\right)$ was used as a measurement of early small airway disease in our study. The correlation between $\mathrm{FEF}_{50 \%}$ and $\mathrm{FEF}_{25-75 \%}$ was also compared and discussed.

\section{Methods}

\section{Study population}

The participants included in this study were enrolled in the electronic medical databases (EMD) of the Respiratory Division of Zhongshan Hospital, Huashan Hospital, Qingpu Branch of Zhongshan Hospital in Shanghai. A total of 250 subjects were screened, and 173 subjects were excluded due to wrong phone number, unwillingness to participate, smoking or age limitations. Finally, 77 diagnosed CVA patients were enrolled. Inclusion criteria: (1) diagnosed with CVA, (2) 18-70 years of age, (3) FEV1/forced vital capacity $(\mathrm{FVC})>70 \%$, forced expiratory volume in the first second (FEV1) of at least $80 \%$ of the predicted value (FEV1/ FVC $>70 \%$, and FEV1\% $>80 \%$ ), (4) all subjects have less than $12 \%$ improvement in FEV1 after bronchodilator inhalation. Exclusion criteria: (1) asthma exacerbation within the previous 3 months, (2) FEV1/FVC $<70 \%$, (3) FEV1/FVC $>70 \%$ but FEV1\% $<80 \%$, (4) upper respiratory infection within the previous 3 months, (5) severe organ dysfunction (for example, respiratory failure, heart failure, liver or kidney failure), (6) pregnancy, (7) unwillingness to be followed for 1 year, (8) smoker. All participants were diagnosed with CVA according to the definition made by ACCP which include: (1) patients present with cough in isolation; (2) patients with normal routine spirometry and bronchial challenge testing reveals the presence of bronchial hyperresponsiveness; (3) the resolution of cough due to specific antiasthmatic treatment [10].

The participants were interviewed in person at the Respiratory Division of Zhongshan Hospital using a structured questionnaire to obtain information about symptoms, lifestyle, medical history, etc. (Table 1). An asthma history questionnaire inquiring about the chief complaint, cough features, sneezing, runny nose in cold air, sputum properties, family history, allergens, and smoking history was completed at baseline. All the participants provided written informed consent. The protocol (No: B2014-109) was approved by the

Table 1 Characteristics of the study population at baseline

\begin{tabular}{|c|c|c|c|}
\hline & $\begin{array}{l}\text { CVA with low } \\
\text { FEF }_{50 \%} \\
(n=27)\end{array}$ & $\begin{array}{l}\text { CVA } \\
\text { with normal } \\
\operatorname{FEF}_{50 \%}(n=30)\end{array}$ & $\mathrm{p}$ value \\
\hline Age (years) & $43.2 \pm 14.1$ & $41.1 \pm 15.6$ & 0.33 \\
\hline Gender (male: female) & $56 \% / 44 \%$ & $27 \% / 73 \%$ & 0.48 \\
\hline \multicolumn{4}{|l|}{ Medical history } \\
\hline Daytime cough & $11(40.7 \%)$ & $6(20 \%)$ & 0.33 \\
\hline Nighttime cough & $19(70.4 \%)$ & $23(77.6 \%)$ & 0.68 \\
\hline Cough affects sleep & $19(70.4 \%)$ & $15(50.0 \%)$ & 0.57 \\
\hline Chest tightness & $20(74.1 \%)$ & $10(33.3 \%)$ & 0.40 \\
\hline Allergic rhinitis & $17(62.9 \%)$ & $22(73.3 \%)$ & 0.15 \\
\hline Allergic family history & $15(55.6 \%)$ & $15(50.0 \%)$ & 0.27 \\
\hline \multicolumn{4}{|l|}{ Pulmonary function test } \\
\hline FEV1/FVC (\%) & $77.4 \pm 6.06$ & $86.0 \pm 6.12$ & $<0.001$ \\
\hline FEV1\% & $90.77 \pm 11.4$ & $99.8 \pm 13.6$ & 0.97 \\
\hline $\mathrm{FEF}_{50 \%}$ & $62.95 \pm 8.06$ & $85.7 \pm 16.7$ & $<0.001$ \\
\hline \multicolumn{4}{|c|}{ Asthma control assessments } \\
\hline ACT & $17.9 \pm 4.4$ & $18.3 \pm 3.0$ & 0.57 \\
\hline ACQ & $1.79 \pm 0.99$ & $1.43 \pm 0.66$ & 0.14 \\
\hline \multicolumn{4}{|l|}{ Induced sputum } \\
\hline $\begin{array}{l}\text { Total number of cells } \\
\left(10^{5} / \mathrm{mL}\right)\end{array}$ & $5.6 \pm 1.2$ & $6.7 \pm 0.9$ & 0.45 \\
\hline Neutrophils (\%) & $30.5 \pm 6.2$ & $47.2 \pm 10.9$ & 0.67 \\
\hline Eosinophils (\%) & $3.6 \pm 0.2$ & $2.7 \pm 0.4$ & 0.55 \\
\hline Lymphocytes (\%) & $7.8 \pm 0.6$ & $9.2 \pm 0.2$ & 0.23 \\
\hline Macrophages (\%) & $36.4 \pm 4.2$ & $32.3 \pm 3.3$ & 0.78 \\
\hline
\end{tabular}


institutional review board at Fudan University prior to the study.

\section{Asthma control assessments}

The Asthma Control Test (ACT) and the Asthma Control Questionnaire (ACQ), designed to assess asthma management, were implemented at baseline (v1 visit) and the follow-up conducted 12 months after baseline (v2 visit). The ACT was rated on a scale of $0-25$, where scores of 25 demonstrated complete asthma control, 20-25 signified good asthma control (well-controlled asthma), and scores below 20 indicated that the patient's disease was not controlled. The ACQ was scored on a scale of 0 to 6 for each question, where 0 represented good control and 6 represented very poor control. There were seven questions in the questionnaire for total score of 42 [11]. The validity and reproducibility of the ACT and ACQ have been described elsewhere $[12,13]$.

\section{Pulmonary function test}

Spirometry and plethysmographic measurements were performed using standard techniques according to ATSERS recommendations. All spirometry was performed by well-trained technicians, and all measurements were repeated three times. Interpretative strategies for lung function tests were previously established and subsequently extended.

\section{Induced sputum}

All participants underwent sputum induction at the first visit. An aerosol of hypertonic saline at a 3\% concentration was generated by an ultrasonic nebulizer. Sputum was induced by three consecutive nebulization sessions that lasted $7 \mathrm{~min}$ each time. After the sputum bolt was picked out and incubated with DTT, the cells were suspended with PBS. The cell suspension was sent to the lab and stained by hematoxylin-eosin (HE) staining.

\section{Follow-up}

The cohort members were followed for asthma management with in-person follow-up surveys administered 12 months later. The follow-up visit included a history review and physical examination, the $\mathrm{ACT} / \mathrm{ACQ}$, and lung function testing. Medications and treatment duration were also recorded for all study participants.

\section{Statistical analysis}

Continuous variables including lung function parameters, ACT/ACQ score, and induced sputum results were tested with a two-sample t-test. The Chisquare test used for classification variables such as sex and age. All statistical analyses were performed using SPSS software, version 21 (IBM Corporation, Somers, NY, USA). All p values were calculated by two-sided tests and were considered statistically significant if $\mathrm{p}$ was less than 0.05 .

\section{Results}

$\mathrm{FEF}_{50 \%}$ is a feasible parameter for identifying small airway dysfunction early

We excluded the total of 173 participants identified through the EMD, in which wrong phone numbers (80 participants), unwillingness to participate (40 participants), age other than 18-70 years (16 participants), patients with proximal airway obstruction (FEV1/FVC $<70 \%$ or $\mathrm{FEV} \%<80 \%$ ) (32 participants), smoker (5 participants). After these exclusions, a total of 77 participants remained in the study. The participants were divided into two groups according to $\mathrm{FEF}_{50 \%}$, which reflects small airway function: $35(45.4 \%)$ were in the lower $\mathrm{FEF}_{50 \%}$ group (the group with small airway disease) and $42(54.6 \%)$ were in the normal $\mathrm{FEF}_{50 \%}$ group (the group with normal small airways). Twenty participants dropped out during follow-up. Finally, 57 were included in the analysis, with 27 in the lower $\mathrm{FEF}_{50 \%}$ group and 30 in the normal $\mathrm{FEF}_{50 \%}$ group (Fig. 1).

The baseline characteristics of the participants are presented in Table 1. The participants in the lower $\mathrm{FEF}_{50 \%}$ group had lower FEV1/FVC values than those in the normal $\mathrm{FEF}_{50 \%}$ group (Fig. 2a). No differences

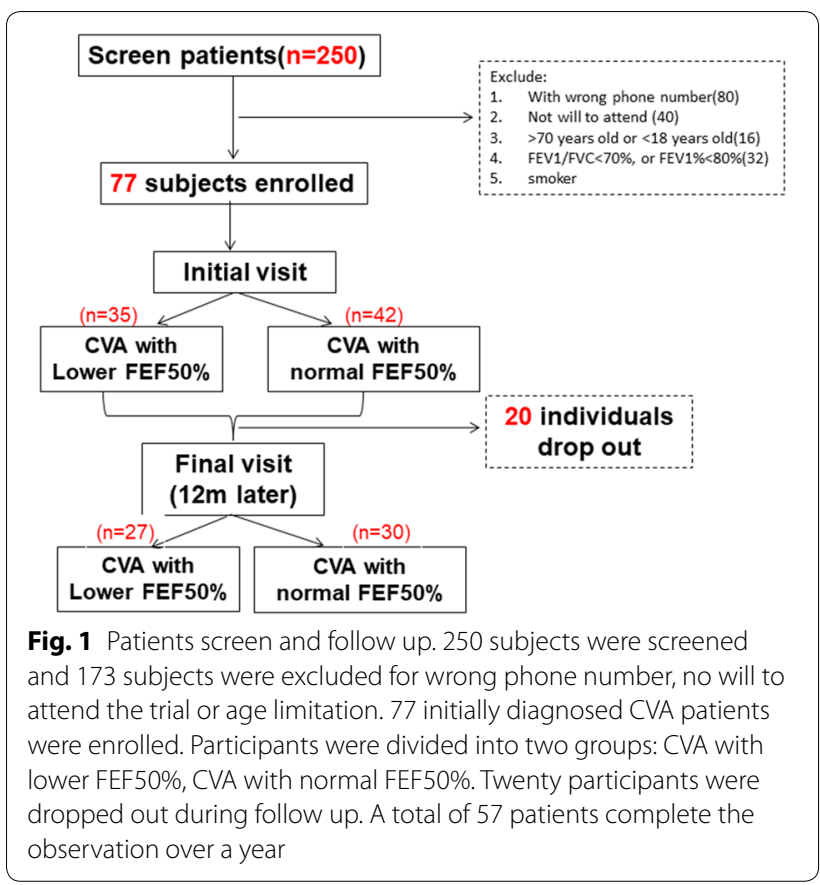



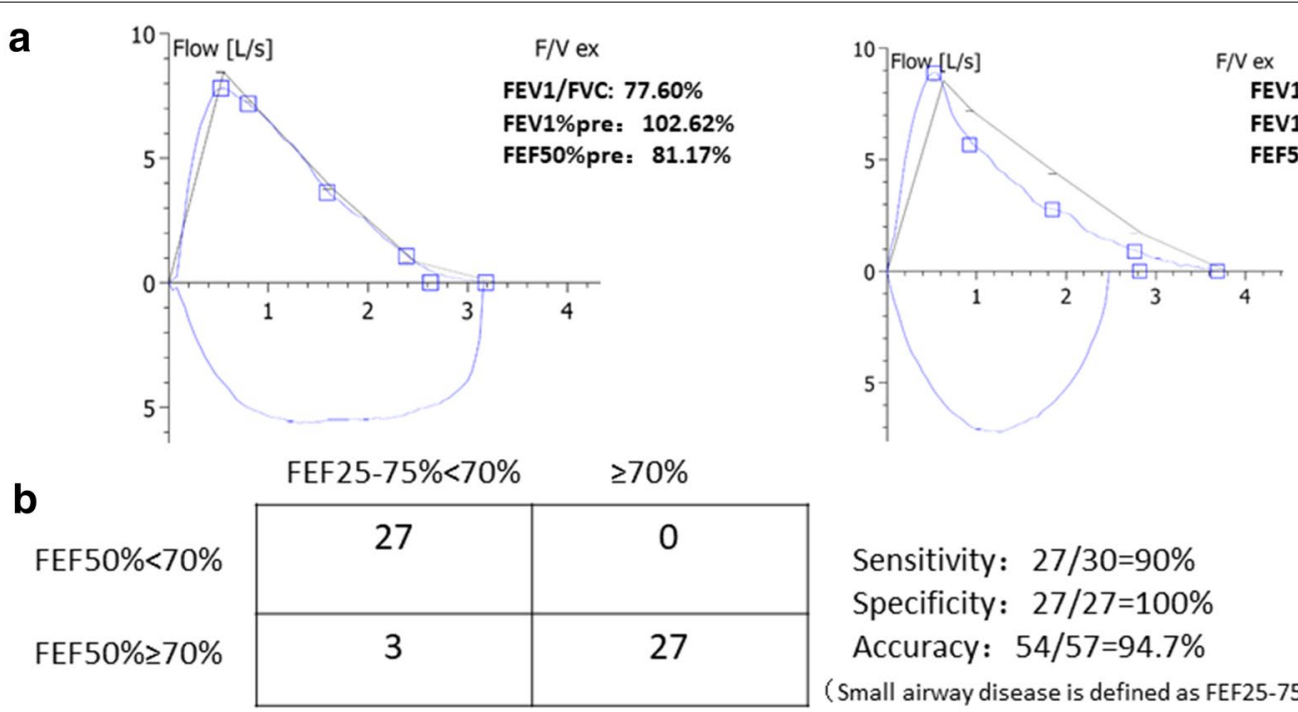

FEV1/FVC: $72.18 \%$

FEV1\% pre: $95.37 \%$

FEF50\%pre: $62.34 \%$

Sensitivity: $27 / 30=90 \%$

Specificity: $27 / 27=100 \%$

Accuracy: $54 / 57=94.7 \%$

( $S$ mall airway disease is defined as FEF $25-75 \%<70 \%$ )

C
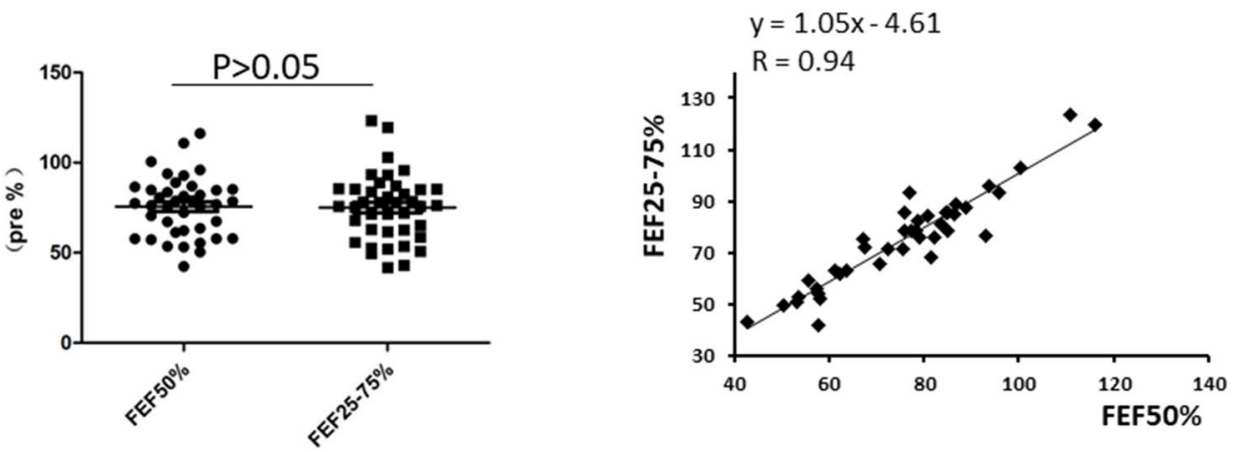

Fig. 2 FEF50\% and FEF25-75\% as early markers of small airway disease. a Representative spirometry results in our cohort are showed. Normal airway is the group CVA with normal FEF50\%. Small airway disease is the group CVA with lower FEF50\%. b Diagnostic test of FEF50\%. FEF25-75\% is defined as gold standard and FEF $25-75 \%<70 \%$ predicted is set as cut value. The sensitivity, specificity and accuracy of new measurement (FEF50\%) are measured. c The average value of FEF50\% and FEF25-75\% ( $p>0.05)$. The linear correlation analysis of FEF50\% and FEF25-75\% in CVA patients. All spirometries were performed by well-trained technician with all data repeated three times

in symptoms, allergy histories, $\mathrm{ACT} / \mathrm{ACQ}$, or induced sputum results between the two groups were observed.

The clinical characteristics, airway eosinophils and drug responsiveness are quite similar in patients with and without small airway disease (Table 1); consequently, more sensitive parameters are urgently needed to identify small airway disease early. $\mathrm{FEF}_{25-75 \%}$ has been shown to be acceptable parameter to evaluate small airway disease [14]. However, it has not been fully accepted by American Thoracic Society (ATS) for determining small airway disease because of its variability compared with FEV1. Thus, we hypothesized that $\mathrm{FEF}_{50 \%}$ may be a substantial parameter with better feasibility. Using $\mathrm{FEF}_{25-75 \%}$ as the ATS old standard, we examined the methodology of $\mathrm{FEF}_{50 \%}$ as a diagnostic test for small airway impairment in CVA patients. We found a sensitivity of $90 \%$ and a specificity of $100 \%$. The accuracy was $94.7 \%$ (Fig. 2b). The mean values of $\mathrm{FEF}_{50 \%}$ and $\mathrm{FEF}_{25-75 \%}$ were $74.12 \% \pm 2.02 \%$ and $75.89 \% \pm 2.1 \%$, respectively $(\mathrm{p}>0.05)$. The correlation coefficient was 0.94 . The extremely close mean values and good correlation prompted us to conclude that $\mathrm{FEF}_{50 \%}$ is an acceptable spirometric parameter for distinguishing small airway dysfunction from normal small airway function. Furthermore, the regression equation was $y=1.05 x-4.61$ (Fig. 2c).

\section{Clinical characteristics of CVA with small airway disease over a 1-year period}

The ACT/ACQ scores showed little difference between the two groups at baseline, indicating that the patients in both groups had similar symptoms and poor control (Table 1). During the follow-up interview, we found that the ACT scores increased significantly in the lower $\mathrm{FEF}_{50 \%}$ group, indicating that CVA improved from not controlled to well controlled $(17.9 \pm 4.5$ to $22.1 \pm 2.6)$. 
There was a trend of improvement of ACT scores in the normal $\mathrm{FEF}_{50 \%}$ group (18.6 \pm 3.2 to $20.4 \pm 5.0$ ), however it didn't reach a statistical significance. The ACQ score decreased in both groups, both of which reach a statistical significance (Fig. 3).

The significant improvement of asthma control in the lower $\mathrm{FEF}_{50 \%}$ group prompted us to further investigate medication usage throughout the follow-up period. We found that $64.6 \%$ of the patients in the lower $\mathrm{FEF}_{50 \%}$ group insisted on regular medication more than 1 month, while $59.2 \%$ in the normal $\mathrm{FEF}_{50 \%}$ group. Obviously, the patients in the lower $\mathrm{FEF}_{50 \%}$ group tended to use bronchodilator and ICS more frequently, especially they used more ICS than control group $(83.1 \%$ to $67.3 \%, \mathrm{p}=0.04$ ) (Fig. 4). We presumed "small airway dysfunction" in pulmonary function report prompted patients more adherent to treatment. This may explain the apparent improvement of asthma control in the lower $\mathrm{FEF}_{50 \%}$ group. Then, we examined the overall recurrence in the two groups. Although it can get good clinical control, the recurrence rate in the lower $\mathrm{FEF}_{50 \%}$ group was much higher than that in the normal $\mathrm{FEF}_{50 \%}$ group $(-75 \% \pm 7.6 \%$ vs $54 \% \pm 4.9 \%$, p < 0.05$)$ (Fig. 5).

\section{Lung function changes in CVA with small airway disease over a 1-year period}

To investigate the lung function changes in CVA patients with small airway disease, we further compared the parameters of expiratory flow, including FEV1, FEV1/ $\mathrm{FVC}$ and $\mathrm{FEF}_{50 \%}$. FEV1/FVC and FEV1\%, which represent proximal airway function, were slightly improved or almost the same over the 1-year study period (Fig. 6). The same was true of $\mathrm{FEF}_{50 \%}$. When we compared the $\mathrm{FEF}_{50 \%}$ between the two groups at the end of the year, the value was still much lower in the CVA with small airway disease group compared with the group with normal small airways $(57.2 \% \pm 10.5 \%$ vs $92.6 \% \pm 16.5 \%)$. The results indicated that the more regular use of anti-asthma medication could improve clinical symptoms, but not small airway obstruction.

\section{Discussion}

Spirometry is the most frequently used, non-invasive way to assess the airflow limitation in asthma. Parameters such as FEV1 (forced expiratory volume at $1 \mathrm{~s}$ ) and FEV1/FVC (the ratio of forced expiratory volume at $1 \mathrm{~s}$ to forced vital capacity) are widely used to evaluate proximal airway obstruction. The forced mid-expiratory flow between 25 and $75 \%\left(\mathrm{FEF}_{25-75 \%}\right)$ can decrease more steeply than the ratio of FEV1/FVC when airway closure and air trapping occur in the distal airways $[15,16]$. Back to 1975, Dosman and colleagues demonstrated $\mathrm{FEF}_{50}$ $\left(\mathrm{V}_{\max 50}\right)$ reduction is a sensitive measure to distinguish small airway obstruction while breathing helium-oxygen mixture especially in smokers [17]. Also, $\mathrm{FEF}_{25-75 \%}$ has been identified as an early marker of small airway impairment in subjects with allergic rhinitis [14]. Several studies have shown a good correlation between $\mathrm{FEF}_{25-}$ $75 \%$ and the HRCT finding of air trapping. Nevertheless, the cut-point of $\mathrm{FEF}_{25-75 \%}$ for evaluating small airway impairment has been controversial. Marseglia et al. [18] defined the cut-point as $<80 \%$ of predicted. Of the 58 subjects with a normal FEV1, FVC, and FEV1/FVC ratio, 20 (34\%) had a reduced $\mathrm{FEF}_{25-75 \%}$ with a mean value $70.3 \pm 8.5$, compared with $108.0 \pm 14.3$ in the patients with preserved $\mathrm{FEF}_{25-75 \%}$. Manoharan et al. [19] selected a cut-point of $60 \%$ of predicted for $\mathrm{FEF}_{25-75 \%}$ to define the presence of small airway disease. A total of 238 patients (54\%) had values $<60 \%$.

In our study, the correlation between $\mathrm{FEF}_{50 \%}$ and $\mathrm{FEF}_{25-75 \%}$ in the CVA population was first evaluated. The $\mathrm{FEF}_{50 \%}$ is an instantaneous flow representing the
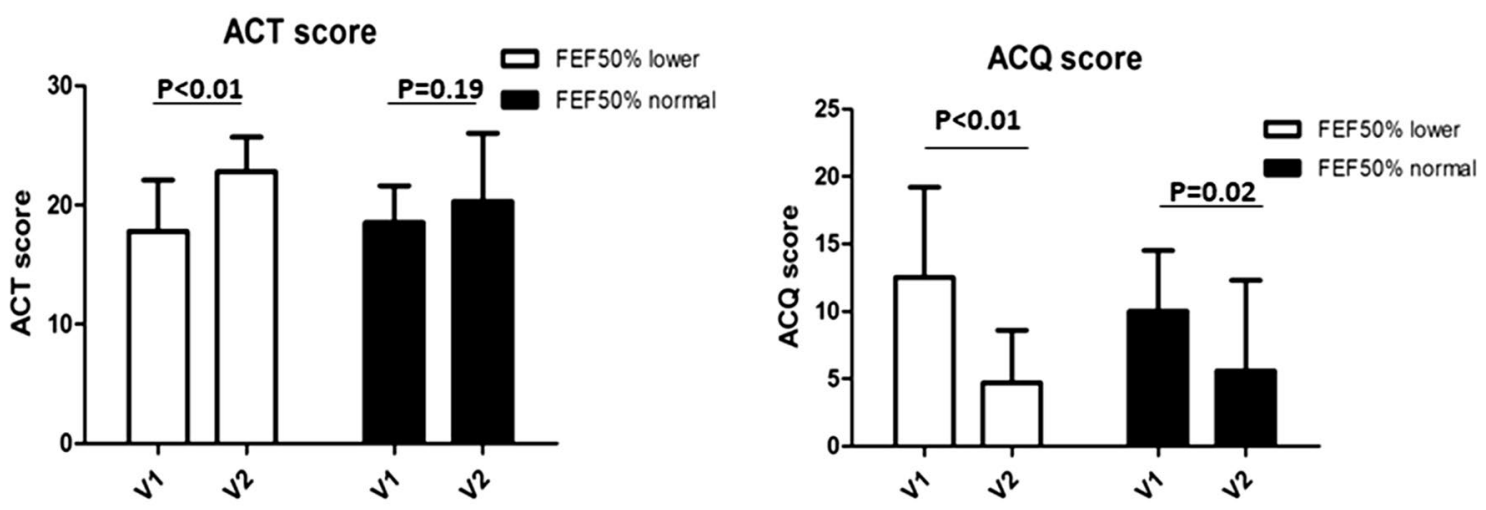

Fig. 3 Asthma clinical control over a year by ACT and ACQ questionnaire. Both ACT and ACQ score improved in both groups when compared final visit with initial visit. The scale of improvement in CVA with lower FEF50\% is much greater than the control groups 


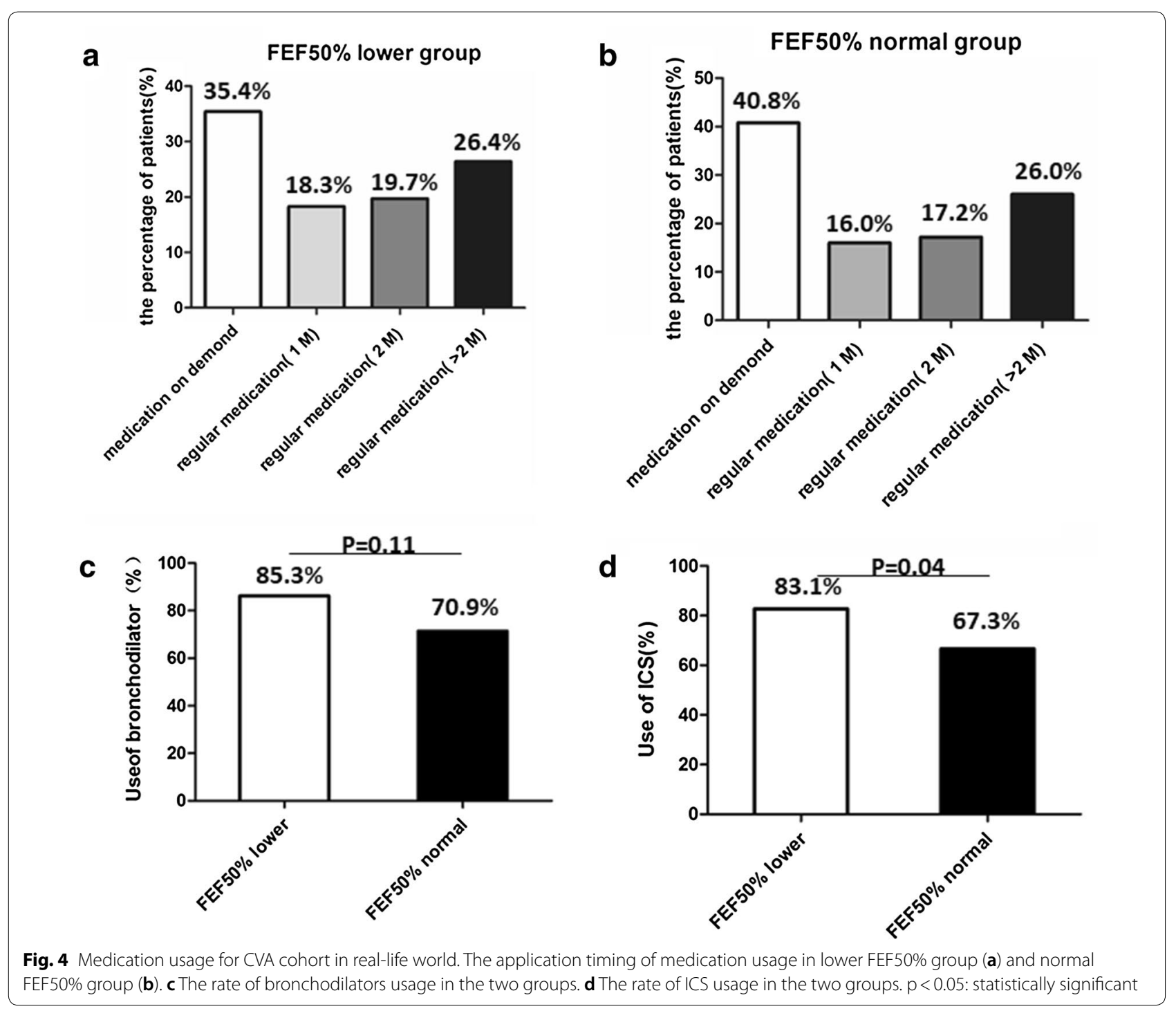

flow rate at half of expiration, whereas the $\mathrm{FEF}_{25-75 \%}$ is an average value over the mid-vital capacity range [20]. When the cut-point of reduced $\mathrm{FEF}_{50 \%}$ was defined as $<60 \%$ predicted, 21 out of 57 subjects (36.8\%) had a reduced $\mathrm{FEF}_{50 \%}$, and the exact same number and proportion were found when the cut-point of reduced $\mathrm{FEF}_{25-75 \%}$ was defined as $<60 \%$. Whether $\mathrm{FEF}_{50 \%}$ predicted or $\mathrm{FEF}<60 \%$ predicted were defined as markers of small airway disease, the judgment of the disease was $100 \%$ consistent between the two indices (the values of average $\mathrm{FEF}_{50 \%}$ or $\mathrm{FEF}_{25-75 \%}$ were $53.9 \%$ and $50.9 \%$, respectively). However, the incidence of small airway disease in our CVA population was relatively low according to this cut-point. We attempted to reset the cut-point of $\mathrm{FEF}_{25-75 \%}<70 \%$ as a measure of small airway disease and found that 30 out of 57 subjects $(52.6 \%)$ had a reduced $\mathrm{FEF}_{25-75 \%} ; 28$ subjects' $\mathrm{FEF}_{50 \%}$ values were also $<70 \%$ of predicted, and 2 patients' values were $>70 \%$ of predicted. If $\mathrm{FEF}_{50 \%}<70 \%$ predicted or $\mathrm{FEF}_{25-}$ $75 \%<70 \%$ predicted are defined as measurements for classifying small airway disease, more patients with early small airway disease will be identified. If the $\mathrm{FEF}_{25-75 \%}$ measurement is defined as ATS old standard, the new indices of $\mathrm{FEF}_{50 \%}$ sensitivity, specificity and accuracy are $90 \%, 100 \%$ and $94.7 \%$. Yuan et al. [21] found that $\mathrm{FEF}_{50 \%}$ was approximately $15 \%$ higher than $\mathrm{FEF}_{25-75 \%}$; furthermore, the difference between the two was fairly constant and was well-preserved in cases of irregularly shaped curves. In our study, the value of $\mathrm{FEF}_{50 \%}$ was $6 \%$ higher than that of $\mathrm{FEF}_{25-75 \%}$ (58.9\% vs $55.6 \%$ ) if $<70 \%$ predicted was used as a measurement of small airway disease, which is consistent with previously published data. Compared with $\mathrm{FEF}_{25-75 \%}, \mathrm{FEF}_{50 \%}$ is simple, easy to understand and does not need to be computed. 


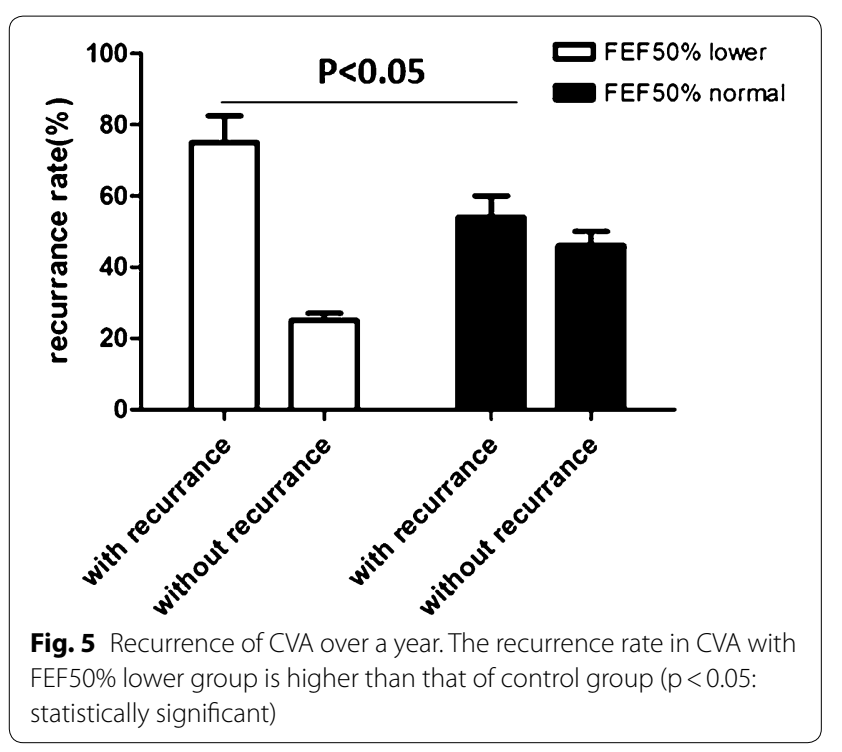

$\mathrm{FEF}_{25-75 \%}$ has been considered as an index of small airway impairment before. However, the ATS guidelines on lung function testing do not support the use of $\mathrm{FEF}_{25-75 \%}$ to identify small airway disease [22]. $\mathrm{FEF}_{25-}$ $75 \%$ is considered more variable than FEV1 because it is influenced by changes in lung volume and the shape of the flow-volume loop. The weakness is that the value should be varied when a series of spirometry are done in the same individual. In our study, all the CVA patients had preserved proximal lung function (with normal FEV1, FVC and FEV1/FVC). All spirometry was performed by well-trained technicians, and all measurements were repeated three times. Therefore, we suggest that in patients with preserved FEV1, $\mathrm{FEF}_{25-75 \%}$ or $\mathrm{FEF}_{50 \%}$ may be used as marker of early small airway disease.

We used $\mathrm{FEF}_{50 \%}<70 \%$ as a marker for small airway disease and found that nearly half of the CVA patients with preserved FEV1 had small airway problems

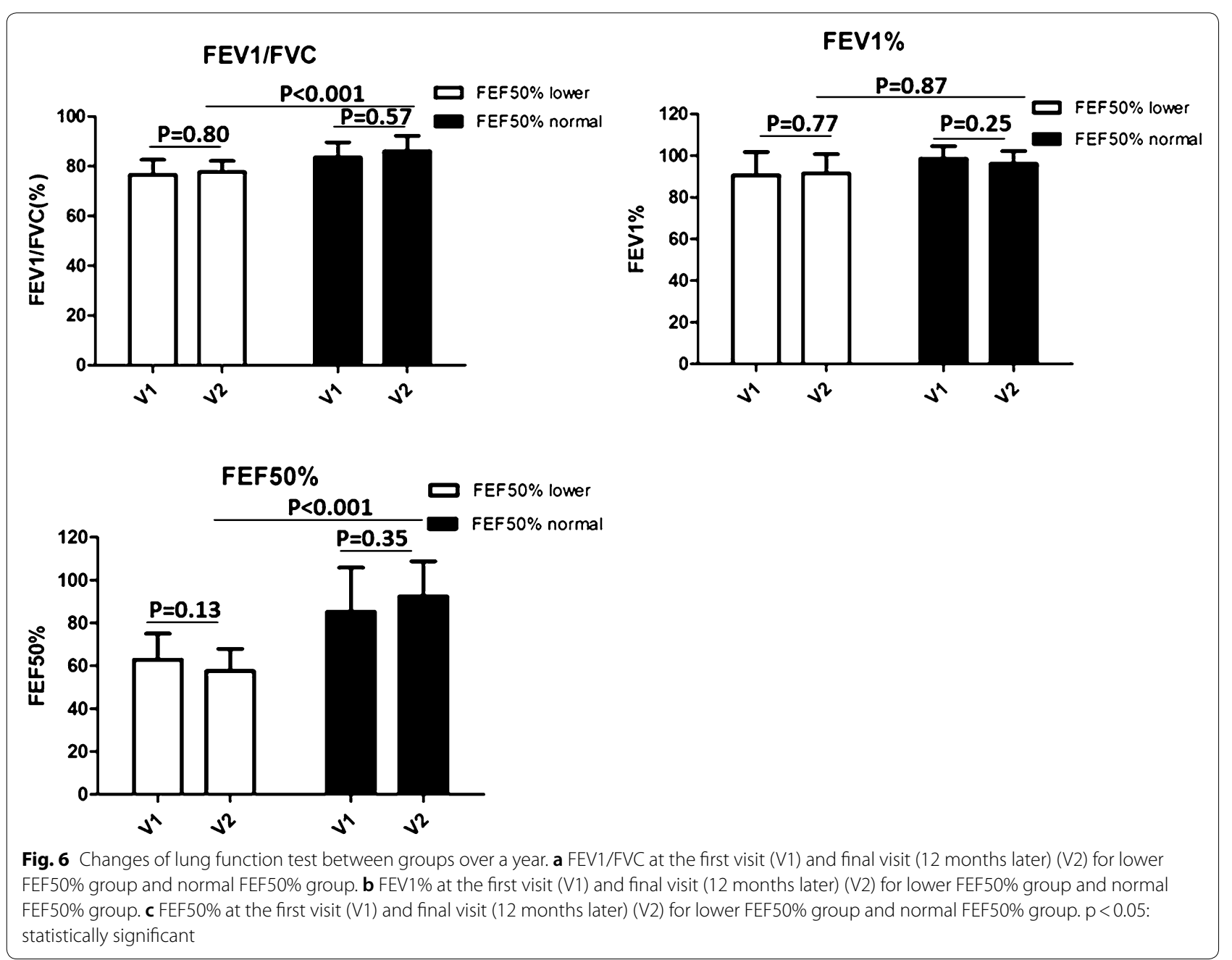


$(35 / 77=45.4 \%)$, which is similar to the $50-60 \%$ prevalence of small airway disease in adult asthma reported by Usmani [7] in a systemic review. It is noteworthy that the clinical symptoms, allergic disease history and inflammation in induced sputum were almost the same between the CVA with lower $\mathrm{FEF}_{50 \%}$ group and the CVA with normal $\mathrm{FEF}_{50 \%}$ group, whereas the $\mathrm{FEF}_{50 \%}$ values differed significantly (62.95\% vs $\left.85.7 \%\right)$. In a study of 58 patients with rhinitis, Marseglia et al. [18] demonstrated that the proportion of subjects with reduced $\mathrm{FEF}_{25-75 \%}$ appeared to increase with increasing severity of allergic predictors (rhinitis symptoms, rhinitis eosinophils and BHR). The more severe the allergy score was, the lower the mean $\mathrm{FEF}_{25-75 \%}$ value appeared to be. However, in our CVA cohort, a link between clinical scores, airway inflammation and the $\mathrm{FEF}_{50 \%}$ value was not observed. The underlying pathogenesis of early small airway dysfunction in CVA patients requires further investigation.

Anderson et al. [23] determined the prevalence of small airway disease in populations with differing asthma severities. Denlinger et al. [24] showed that small airway dysfunction, measured by $\mathrm{FEF}_{25-75 \%}$, was positively correlated with exacerbation frequency in both adults and children with exacerbation-prone asthma. Similarly, children from a Boston cohort with reduced $\mathrm{FEF}_{25-75 \%}$ had a substantially higher risk of exacerbations and systemic steroid use compared with children with normal lung function [25]. Our cohort study not only illustrated the prevalence of small airway disease in one subtype of mild asthma-CVA-but also observed the outcome of CVA with small airway disease over a 1-year period. The symptom scores (ACT and ACQ) at follow-up in CVA patients with small airway disease improved markedly compared with the initial visit. Interestingly, the changes in scores (ACT and ACQ) were even greater in the CVA with small airway disease group than in the CVA with normal small airways group. Lung function tests were performed at both the initial and follow-up visits; while parameters of expiratory flow such as FEV1, FEV1/FVC and $\mathrm{FEF}_{50 \%}$ improved slightly or were almost the same after 1 year in both groups, the recurrence rate was significantly higher in the CVA with small airway disease group compared with the CVA with normal small airways group. A team of multinational clinicians initiated a study called the longitudinal assessment of small airways involvement in asthma (ATLANTIS) in 2014. They plan to include 900 subjects and follow them for 1 year. All small airway diseases will be evaluated by spirometry, MBNW, IOS, and CT scan. We await the results of this study to optimize our knowledge of the prevalence and prognosis of asthma with small airway disease [26].
In the cohort, the participants received therapy according to standard clinical care, without any further pharmacological intervention. For the CVA patients with small airway disease, the $\mathrm{FEF}_{50 \%}$ value was almost unchanged after 1 year and was still much lower than the mean $\mathrm{FEF}_{50 \%}$ in CVA patients with normal small airways $(57.2 \% \pm 10.5 \%$ vs $92.6 \% \pm 16.5 \%)$. Interestingly, the asthma control status at the final visit was better in the CVA patients with small airway disease than in those without small airway disease. This is probably due to more regular and longer medication usage in real life among CVA patients with small airway disease. We presume that the notation of "small airway dysfunction" in the lung function report prompted these patients to adhere to treatment and regular check-ups. We found that the ICS-sold (for example budesonide) on the Chinese market did not improve small airway dysfunction over a year. Whether the newly developed extra-fine particle ICS (beclomethasone/formoterol, propelled by hydrofluoroalkane) has the ability to improve small airway disease? It is reported to have a mean aerodynamic diameter of $1-2 \mu \mathrm{m}$ and can reach small airways, leading to an increase in the lung deposition rate as high as 50-68\% [27-29]. Thus, the efficacy of extra-fine particle ICS for CVA patients with small airway disease requires further investigation.

\section{Conclusion}

In conclusion, we found that nearly half of our CVA population had small airway disease. Their demographic features, clinical characteristics, airway eosinophil levels and drug responsiveness were quite similar to those of patients without small airway disease. CVA with small airway disease has higher recurrence rate after anti-asthmatic therapy. $\mathrm{FEF}_{50 \%}$ is suggested as an easy and feasible marker for the early identification of this subpopulation with small airway disease. Unlike FEV1\% as a marker of proximal airway obstruction, small airway disease assessments are in their relative infancy, and population-based data are urgently needed. Other techniques, such as MBNW, IOS, alveolar FeNO and micro $\mathrm{CT}$, are also being used to evaluate small airway disease [29-32]. The strengths and shortcomings of different methods will be compared in the future [16, $33,34]$. Longitudinal studies with larger populations are needed to determine whether ultrafine particles affect the outcome of CVA in terms of asthma control, lung function improvement and disease recurrence.

\section{Abbreviations}

ACQ: Asthma Control Questionnaire; ACT: Asthma Control Test; ATLANTIS: longitudinal assessment of small airways involvement in asthma; ATS: 
American Thoracic Society; CVA: cough variant asthma; EMD: electronic medical databases; FEV1: forced expiratory volume in $1 \mathrm{~s}$; $\mathrm{FEF}_{50 \%}$ : forced expiratory flow at 50\%; FEF25-75\%: forced mid-expiratory flow between 25 and 75\%; FVC: forced vital capacity; HE: hematoxylin-eosin; HRCT: highresolution computerized tomography.

\section{Acknowledgements}

This work's design and statistical analysis were instructed by ATS MECOR training programme (Pro Sonia Buist, Pro Shannon Carson and Pro David Au).

\section{Authors' contributions}

Conceived and designed the study: ZC, CD and GW. Performed the biological experiments: HY, XL, LL. Statistical analysis: $C L, Y Z$ and RM. Wrote the paper: $H Y$, $\mathrm{XL}$ and $\mathrm{ZC}, \mathrm{CD}$. All authors read and approved the final manuscript.

\section{Funding}

This work was supported by National Natural Science Foundation of China $(81270078,81470211$ by ZHC) and Shanghai 3 year Plan of the Key Subjects Construction in Public Health-Infectious Disease and Pathogenic Microorganism (15GWZK0102, by LZ), Shanghai Top-Priority Clinical Key Disciplines Construction Project (2017ZZ02013), Shanghai Health Committee (201840288) and research fund from Shanghai Respiratory Research Institute and Yang Scientists training program from Zhongshan Hospital.

\section{Availability of data and materials}

The datasets used and analysed during the current study are available from the corresponding author on reasonable request.

\section{Ethics approval and consent to participate}

All the participants provided written informed consent before entering the study. The approval was renewed for the first and the second follow-up. The protocol (No: B2014-109) was approved by the institutional review board at Fudan University prior to the study.

\section{Consent for publication}

Not applicable.

\section{Competing interests}

The authors declare that they have no competing interests.

\section{Author details}

${ }^{1}$ Respiratory Division of Zhongshan Hospital, Shanghai Institute of Respiratory Disease, Fudan University, No. 180 Fenglin Road, Shanghai, China. ${ }^{2}$ Respiratory Division of the Affiliated Hospital of Qingdao University, Qingdao, China.

${ }^{3}$ Department of Respiratory and Critical Care Medicine, Clinical Research Center for Respiratory Disease, West China Hospital, Sichuan University, Chengdu, China. ${ }^{4}$ Department of Laboratory Medicine, Huashan Hospital, Shanghai Medical College, Fudan University, Shanghai, China. ${ }^{5}$ Respiratory Division of Qingpu Hospital Affiliated to Zhongshan Hospital, Fudan University, Shanghai, China.

Received: 8 January 2019 Accepted: 20 June 2019 Published online: 02 July 2019

\section{References}

1. Baraldo S, Saetta M, Cosio MG. Pathophysiology of the small airways. Semin Respir Crit Care Med. 2003;24:465-72.

2. van der Wiel E, Postma DS, van der Molen T, Schiphof-Godart L, Ten Hacken $\mathrm{NH}$, van den Berge $\mathrm{M}$. Effects of small airway dysfunction on the clinical expression of asthma: a focus on asthma symptoms and bronchial hyper-responsiveness. Allergy. 2014;69:1681-8.

3. Petsonk EL, Stansbury RC, Beeckman-Wagner LA, Long JL, Wang ML. Small airway dysfunction and abnormal exercise responses. A study in coal miners. Ann Am Thorac Soc. 2016;13:1076-80.

4. Hogg JC, Pare PD, Hackett TL. The contribution of small airway obstruction to the pathogenesis of chronic obstructive pulmonary disease. Physiol Rev. 2017;97:529-52.

5. Scichilone N, Contoli M, Paleari D, Pirina P, Rossi A, Sanguinetti CM, Santus P, Sofia M, Sverzellati N. Assessing and accessing the small airways; implications for asthma management. Pulm Pharmacol Ther. 2013;26:172-9.

6. Gonem S, Natarajan S, Desai D, Corkill S, Singapuri A, Bradding P, Gustafsson P, Costanza R, Kajekar R, Parmar H, et al. Clinical significance of small airway obstruction markers in patients with asthma. Clin Exp Allergy. 2014;44:499-507.

7. Usmani OS, Singh D, Spinola M, Bizzi A, Barnes PJ. The prevalence of small airways disease in adult asthma: a systematic literature review. Respir Med. 2016;116:19-27.

8. van den Berge M, ten Hacken NHT, Cohen J, Douma WR, Postma DS. Small airway disease in asthma and COPD: clinical implications. Chest. 2011;139:412-23.

9. Fujimura M. Pathophysiology, diagnosis and treatment of cough variant asthma. Rinsho Byori. 2014;62:464-70.

10. Dicpinigaitis PV. Chronic cough due to asthma: ACCP evidence-based clinical practice guidelines. Chest. 2006;129:75S-9S.

11. Voorend-van Bergen S, Vaessen-Verberne AA, de Jongste JC, Pijnenburg MW. Asthma control questionnaires in the management of asthma in children: a review. Pediatr Pulmonol. 2015;50:202-8.

12. Jia CE, Zhang HP, LV Y, Liang R, Jiang YQ, Powell H, Fu JJ, Wang L, Gibson PG, Wang G. The asthma control test and asthma control questionnaire for assessing asthma control: systematic review and meta-analysis. J Allergy Clin Immunol. 2013;131:695-703.

13. Konstantinos Katsoulis K, Kostikas K, Kontakiotis T. Techniques for assessing small airways function: possible applications in asthma and COPD. Respir Med. 2016;119:e2-9.

14. Yanai M, Sekizawa K, Ohrui T, Sasaki H, Takishima T. Site of airway obstruction in pulmonary disease: direct measurement of intrabronchial pressure. J Appl Physiol. 1985;1992(72):1016-23.

15. in 't Veen JC, Beekman AJ, Bel EH, Sterk PJ. Recurrent exacerbations in severe asthma are associated with enhanced airway closure during stable episodes. Am J Respir Crit Care Med. 2000;161:1902-6.

16. Perez T, Chanez P, Dusser D, Devillier P. Small airway impairment in moderate to severe asthmatics without significant proximal airway obstruction. Respir Med. 2013;107:1667-74.

17. Dosman J, Bode F, Urbanetti J, Martin R, Macklem PT. The use of a helium-oxygen mixture during maximum expiratory flow to demonstrate obstruction in small airways in smokers. J Clin Invest. 1975;55:1090-9.

18. Marseglia GL, Cirillo I, Vizzaccaro A, Klersy C, Tosca MA, La Rosa M, Marseglia A, Licari A, Leone M, Ciprandi G. Role of forced expiratory flow at $25-75 \%$ as an early marker of small airways impairment in subjects with allergic rhinitis. Allergy Asthma Proc. 2007;28:74-8.

19. Manoharan A, Anderson WJ, Lipworth J, Lipworth BJ. Assessment of spirometry and impulse oscillometry in relation to asthma control. Lung. 2015;193:47-51.

20. Cirillo I, Klersy C, Marseglia GL, Vizzaccaro A, Pallestrini E, Tosca M, Ciprandi G. Role of FEF $25 \%-75 \%$ as a predictor of bronchial hyperreactivity in allergic patients. Ann Allergy Asthma Immunol. 2006;96:692-700.

21. Yuan J, An SH, Gao WJ, Du WJ, Sun JF, Zhang M, Yao CZ. Comparative analysis of conventional pulmonary function test results in children with asthma or cough variant asthma. Zhongguo Dang Dai Er Ke Za Zhi. 2013;15:171-4.

22. Usmani OS, Barnes PJ. Assessing and treating small airways disease in asthma and chronic obstructive pulmonary disease. Ann Med. 2012;44:146-56.

23. Anderson WJ, Zajda E, Lipworth BJ. Are we overlooking persistent small airways dysfunction in community-managed asthma? Ann Allergy Asthma Immunol. 2012;109(185-189):e182.

24. Denlinger LC, Phillips BR, Ramratnam S, Ross K, Bhakta NR, Cardet JC, Castro M, Peters SP, Phipatanakul W, Aujla S, et al. Inflammatory and comorbid features of patients with severe asthma and frequent exacerbations. Am J Respir Crit Care Med. 2017;195:302-13.

25. Rao DR, Gaffin JM, Baxi SN, Sheehan WJ, Hoffman EB, Phipatanakul W. The utility of forced expiratory flow between $25 \%$ and $75 \%$ of vital capacity in predicting childhood asthma morbidity and severity. J Asthma. 2012:49:586-92.

26. Postma DS, Brightling C, Fabbri L, van der Molen T, Nicolini G, Papi A, Rabe KF, Siddiqui S, Singh D, van den Berge M, Kraft M. Unmet needs for the assessment of small airways dysfunction in asthma: introduction to the ATLANTIS study. Eur Respir J. 2015;45:1534-8. 
27. Kemp L, Haughney J, Barnes N, Sims E, von Ziegenweidt J, Hillyer EV, Lee AJ, Chisholm A, Price D. Cost-effectiveness analysis of corticosteroid inhaler devices in primary care asthma management: a real world observational study. Clinicoecon Outcomes Res. 2010;2:75-85.

28. Boulet LP, Bateman ED, Voves R, Muller T, Wolf S, Engelstatter R. A randomized study comparing ciclesonide and fluticasone propionate in patients with moderate persistent asthma. Respir Med. 2007;101:1677-86.

29. Beam DS. Value of inhaled corticosteroid therapy in long-term asthma management. Pharm Ther. 2010;35:377-416.

30. Lipworth B, Manoharan A, Anderson W. Unlocking the quiet zone: the small airway asthma phenotype. Lancet Respir Med. 2014;2:497-506.

31. Kjellberg S, Houltz BK, Zetterstrom O, Robinson PD, Gustafsson PM. Clinical characteristics of adult asthma associated with small airway dysfunction. Respir Med. 2016;117:92-102.
32. Schneider A, Faderl B, Schwarzbach J, Welker L, Karsch-Volk M, Jorres RA. Prognostic value of bronchial provocation and FENO measurement for asthma diagnosis-results of a delayed type of diagnostic study. Respir Med. 2014;108:34-40.

33. Carr TF, Altisheh R, Zitt M. Small airways disease and severe asthma. World Allergy Organ J. 2017;10:20.

34. McDonough JE, Yuan R, Suzuki M, Seyednejad N, Elliott WM, Sanchez PG, Wright AC, Gefter WB, Litzky L, Coxson HO, et al. Small-airway obstruction and emphysema in chronic obstructive pulmonary disease. N Engl J Med. 2011;365:1567-75.

\section{Publisher's Note}

Springer Nature remains neutral with regard to jurisdictional claims in published maps and institutional affiliations.
Ready to submit your research? Choose BMC and benefit from:

- fast, convenient online submission

- thorough peer review by experienced researchers in your field

- rapid publication on acceptance

- support for research data, including large and complex data types

- gold Open Access which fosters wider collaboration and increased citations

- maximum visibility for your research: over 100M website views per year

At BMC, research is always in progress.

Learn more biomedcentral.com/submissions 